\title{
Cuban Research in Current International Journals
}

The following selection-alphabetical by title-reflects Cuban publishing in international medical and population health journals over the last quarter on an array of topics. Links to these journal articles may be found at www.medicc.org/mediccreview.

Access to antiepileptic drug therapy in children in Camagüey Province, Cuba. Bárzaga Z, López A, Mejías Y, González AR, Acosta M, Carbonell D, et al. Int J Pharmacy Practice. 2012 May 21. Epub ahead of print.

Objective To describe access to antiepileptic drug therapy and estimate the prevalence of epilepsy in children in Camagüey Province, Cuba. Methods All the community pharmacies in the province were visited and information collected about the number of children receiving antiepileptic drugs in 2009. Availability and cost of each antiepileptic drug were determined. The prevalence of epilepsy was estimated by determining the number of children receiving antiepileptic drugs. Results There were 923 children who received a total of 977 antiepileptic drugs in Camagüey Province. The estimated prevalence of epilepsy was 5.18 per thousand children which is lower than previously reported rates in other low and lower-middle income countries. Most of the children $(871,94 \%)$ received a single antiepileptic drug. Carbamazepine and valproate were the two most frequently prescribed antiepileptic drugs. Antiepileptic drugs were available from the local pharmacy on $76 \%$ of occasions. If the antiepileptic drug was not available from the local pharmacy, the parent had to travel to another pharmacy to obtain the medicine. Conclusions The estimated prevalence of epilepsy in children in Cuba is lower than that estimated in other lower-middle income countries. Access to drug therapy in children with epilepsy can be achieved in lower-middle income countries.

An SYBR Green-based real-time RT-PCR assay for the detection of $\mathrm{H} 5$ hemagglutinin subtype avian influenza virus. Pérez LJ, Díaz de Arce H, Cilloni F, Salviato A, Marciano S, Perea CL, et al. Mol Cell Probes. 2012 Mar 7.

Increasing diversity among H5 hemagglutinin (HA) subtype avian influenza (Al) viruses has resulted in the need of novel sensitive and specific molecular assays. In this study, an SYBR Green-based real-time reverse transcriptionPCR (RRT-PCR) assay was developed for the detection of $\mathrm{H} 5$ subtype $\mathrm{Al}$ virus. Sequence analysis of the Mexican lineage H5N2 isolates (subgroup B) revealed several mismatches in the primer/hydrolysis probe set reported in the commonly used RRT-PCR assay for the detection of H5 North American lineage. The present assay was designed to circumvent the challenge that these viruses represent for the specific detection of $\mathrm{H} 5$ subtype Al viruses. This RRT-PCR assay successfully detected a range of different $\mathrm{H} 5$ subtype Al strains from both Eurasian and North American lineages represent- ing different avian $\mathrm{H} 5 \mathrm{HA}$ clades from diverse geographical locations. The sensitivity of the present method was determined by using in vitro-transcribed RNA and 10-fold serial dilutions of titrated Al viruses. High sensitivity levels were obtained, with limits of detection of $10(0)$ $50 \%$ egg infectious dose $(\operatorname{EID}(50)) / \mathrm{mL}$ and 4.2 gene copies $/ \mu \mathrm{L}$. The linear ranges of the assay span within 10(6)-10(0) EID(50)/mL and 10(6)$10(0)$ gene copies $/ \mu \mathrm{L}$. The results obtained from this method were directly compared with those of the H5 RRT-PCR assay recommended by the OIE. The comparison was performed with 110 tracheal and cloacal swabs from various bird species collected during field and laboratory investigations in Eurasia and Africa in 2006 and 2008 and showed $100 \%$ agreement. This assay is recommended as an alternative method, also allowing a 'double check' approach detection, to be used mainly in outbreak scenarios with higher risk of poultry infections by Central American/ Caribbean H5 Al viruses.

Cultural factors in primary health care in Cuba. A view from community praxis. Martínez Tena AC. Global J Commun Psychol Practice. 2012 Mar;3(1):124-32.

The changes and transformations in Primary Health Care $(\mathrm{PHC})$ developed in recent years from the implementation of the model of Physician and Nurse of the Family in Cuba, must be supported by a shift in perspective and move from an eminently biologicist paradigm to a biosociocultural paradigm. This makes it possible to explicitly situate the relationship between culture and health and explain the social and cultural factors related to healthy behaviors, and also to support interdisciplinary and transdisciplinary studies. The results presented in this paper corroborate the theoretical postulates of cultural anthropology, cultural sociology and medicine that argue that the gap between multidisciplinary health teams and social actors occurs, among other reasons, because of existing traditional cultural etiological models of health and disease. Cultural differences could be addressed through knowledge of the patient's culture, his cultural practices and his links with family members and other stakeholders in the community. It allows the productions of a process that includes cultural communication, listening carefully [to] the patient's point of view rather than just ordering treatments that reflect scientific practices. The strategies and backgrounds presented in this study demand the presence of multiple perspectives and are very important to designing policies and models of intercultural health care to achieve satisfaction of patients, families and communities.
Erythropoietin: still on the neuroprotection road. Subirós N, Del Barco DG, Coro-Antich RM. Ther Adv Neurol Disord. 2012 May;5(3):161-73.

Acute stroke is one of the major causes of death and disabilities. Since the 1980s many clinical studies have been conducted to evaluate neuroprotective approaches to treat this important brain vascular event. However, to date the only drug approved (recombinant tissue plasminogen activator [rtPA]) represents a thrombolytic, nonneuroprotective approach. An important neuroprotective strategy is based on erythropoietin (EPO). Exogenously administered EPO exhibits neuroprotective effects in numerous animal models, through the activation of antiapoptotic, anti-oxidant and anti-inflammatory pathways as well as through the stimulation of angiogenic and neurogenic events. The capability of EPO to cross the blood-brain barrier after systemic administration and its effective therapeutic window are advantages for human acute stroke therapy. However, a multicenter stroke trial where recombinant human EPO (rhEPO) was combined with rtPA had negative outcomes. The present paper reviews the EPO neuroprotective strategy and its mechanisms in ischemic stroke and in other human nervous system diseases.

High-level expression of Falcipain-2 in Escherichia coli by codon optimization and autoinduction. Sarduy ES, Muñoz AC, Trejo SA, Chavéz Planes MD. Protein Expr Purif. 2012 Mar 17;83(1):59-69.

Falcipain-2, the major cysteine hemoglobinase from the human malaria parasite Plasmodium falciparum, is critical for parasite development and is considered a promising chemotherapeutic target. In order to facilitate the high-throughput screening of Falcipain-2 inhibitors from natural sources, we developed an economic and highlyproductive overexpression system in Escherichia coli using a codon-optimized proFalcipain-2 construct. Very high expression levels (35-55\% of total host proteins) were observed when proFalcaipain-2 expression was induced with $1 \mathrm{mM}$ isopropyl-1-thio- $\beta$-d-galactopyranoside (IPTG) in several $E$. coli strains, with the highest level observed for BL21(DE3). A lower expression ( $40 \%$ of total host proteins) was observed when BL21(DE3) was grown in ZYM-5052 autoinduction medium, containing $0.2 \%$ lactose as inducer. However, the culture grew to notably higher cellular density, increasing $\sim 1.5$ times the overall yield of the system when compared with conventional IPTG-induction. Although several conditions were modified to achieve the expression of soluble and active Falcipain-2, the 
enzyme was mainly obtained in the form of insoluble aggregates. After purification and refolding, $\sim 50 \mathrm{mg}$ of active enzyme were obtained per liter of culture at low cost using a regular incubator shaker, and recombinant Falcipain-2 exhibited structural and functional characteristics very similar to the natural counterpart. Due to its versatility and simplicity, this strategy can be straightforwardly adapted to other proteins from Plasmodium species or any other organism with an AT-rich genome.

Immunopotentiating properties of a multispecific $\alpha$-anti-idiotype antibody. Hernández T, Mateo de Acosta C, Pérez R. AMbs. 2012 May $1 ; 4(3)$.

Multispecificity is not a well-understood property of some antibodies. Different functions have been attributed to multispecific natural antibodies, commonly associated with the neutralization and clearance of antigens. Much less is known about the role of antibodies like these, based on their idiotypic connectivity. B7Y33 is a chimeric IgG1 version of a polyreactive $\alpha$ - antiidiotype antibody that is able to interact with different immunoglobulin and non-immunoglobulin antigens. Here we report the capacity of this antibody to enhance the immunogenicity of several autologous IgMs in adjuvant-free conditions. Our results suggest that the formation of immune complexes seems to be necessary, but not sufficient, to this activity. The potential involvement of the interaction of B7Y33 with the FcyRIlb is discussed.

Maturational changes in the human envelope-following responses. Mijares Nodarse E, Pérez Abalo MC, Torres Fortuny A, Vega Hernández M, Lage Castellanos A. Acta Otorrinolaringol Esp. 2012 Mar 13.

Introduction The auditory ability to discriminate rapid changes in the envelope of language sounds is essential for speech comprehension. Human envelope-following responses (EFRs) are useful for objective measurement of temporal auditory processing in subjects who are unable to give accurate behavioural responses (e.g., young children). Objective To evaluate age-dependent changes in EFRs during the first 2 years of life. Methods The EFRs were recorded in a sample of 16 well babies distributed into 2 age groups (G1: 12 newborns; G2: 4 babies of 2 years). The EFRs were evoked by white noise carrier stimuli with a sweep of modulation frequencies from 20 to $200 \mathrm{~Hz}$ presented at $50 \mathrm{~dB} \mathrm{HL}$. Results The age-related changes affected both morphology and EFR detectability. The main morphological differences were at the expense of frequencies below $50 \mathrm{~Hz}$, where the first component $\mathrm{P} 1$ was not well defined in either of the 2 age groups. For all modulation frequencies, age significantly affected EFR amplitude and detectability. Conclusions The present study provides the first evidence on EFR maturation. Some understanding of normal EFR development would facilitate a better use of this technique in clinically-objective measurement of auditory temporal processing in infants who cannot provide reliable behavioural responses.

Meningococcal disease serogroup C. Dickinson FO, Pérez AE, Cuevas IE. Risk Manag Healthc Policy. 2012;5:1-15.

Despite current advances in antibiotic therapy and vaccines, meningococcal disease serogroup C (MDC) remains a serious threat to global health, particularly in countries in North and Latin America, Europe, and Asia. MDC is a leading cause of morbidity, mortality, and neurological sequelae and it is a heavy economic burden. At the individual level, despite advances in antibiotics and supportive therapies, case fatality rate remains nearly $10 \%$ and severe neurological sequelae are frequent. At the population level, prevention and control of infection is more challenging. The main approaches include health education, providing information to the public, specific treatment, chemoprophylaxis, and the use of vaccines. Plain and conjugate meningococcal $\mathrm{C}$ polysaccharide vaccines are considered safe, are well tolerated, and have been used successfully for over 30 years. Most high-income countries use vaccination as a part of public health strategies, and different meningococcal $C$ vaccination schedules have proven to be effective in reducing incidence. This is particularly so with conjugate vaccines, which have been found to induce immunogenicity in infants (the age group with the highest incidence rates of disease), stimulate immunologic memory, have longer effects, not lead to hyporesponsiveness with repeated dosing, and decrease acquisition of nasopharyngeal carriage, inducing herd immunity. Antibiotics are considered a cornerstone of MDC treatment and must be administered empirically as soon as possible. The choice of which antibiotic to use should be made based on local antibiotic resistance, availability, and circulating strains. Excellent options for a 7-day course are penicillin, ampicillin, chloramphenicol, and third-generation cephalosporins (ceftriaxone and cefotaxime) intravenously, although the latter are considerably more expensive than the others. The use of steroids as adjunctive therapy for MDC is still controversial and remains a topic of debate. A combination of all of the aforementioned approaches is useful in the prevention and control of MDC, and each country should tailor its public health policy to its own particular needs and knowledge of disease burden.

On the safety of persons accompanying nuclear medicine patients. Díaz $M$, López GM, Varela C, Fleitas I. Dosimetry Radiat Prot. 2012 Apr;150(2).

The presence of caretakers/comforters during nuclear medicine examinations is relatively common. These caretakers receive higher doses than the general public, who receive only environmental/background exposure. The aim of this research was to know about the doses received by two significant groups of caretakers: comforters of cancer patients (Group I) and mothers of small children (Group II). The patients were scheduled to undergo two different diagnostic studies: Inmuno-Scintigraphy using a monoclonal antibody bound to ${ }^{99 \mathrm{~m}} \mathrm{Tc}$ (for adults) and Renal Scintigraphy using ${ }^{99 m}$ Tcdimercaptosuccinic acid (for children). The average effective doses were 0.27 and 0.29 $\mathrm{mSv}$ for Groups I and II, respectively. Additionally, environmental monitoring was performed in the waiting room for injected patients (Room I) and inside the procedure room (Room II). Equivalent environmental doses of 0.28 and $0.24 \mathrm{mSv}$ for Rooms 1 and II, respectively, were found, which are similar to values reported by other authors.

Protective effect of D-002, a mixture of beeswax alcohols, against indomethacininduced gastric ulcers and mechanism of action. Pérez Y, Oyárzabal A, Mas R, Molina V, Jiménez S. J Nat Med. 2012 May 11.

D-002, a mixture of higher aliphatic beeswax alcohols, produces gastroprotective and antioxidant effects. To investigate the gastroprotective effect of D-002 against indomethacin-induced ulcers, oxidative variables and myeloperoxidase (MPO) activity in the rat gastric mucosa were examined. Rats were randomized into six groups: a negative vehicle control and five indomethacin (50 $\mathrm{mg} / \mathrm{kg}$ ) treated groups, comprising a positive control, three groups treated orally with D-002 (5, 25 and $100 \mathrm{mg} / \mathrm{kg})$ and one group with omeprazole $20 \mathrm{mg} / \mathrm{kg}$ intraperitoneally (ip). The contents of malondialdehyde (MDA), protein carbonyl groups (PCG), hydroxyl radical generation and catalase (CAT), glutathione peroxidase (GSH-PX), superoxide dismutase (SOD) and MPO enzyme activities in the rat gastric mucosa were assessed. Indomethacin increased the content of MDA and $\mathrm{PCG}$, the generation of ${ }^{*} \mathrm{OH}$ radical and MPO enzyme activity, while it decreased the CAT, GSH-PX and SOD activities as compared to the negative controls. D-002 (5-100 $\mathrm{mg} / \mathrm{kg}$ ) significantly and dose-dependently reduced indomethacin-induced ulceration to $75 \%$. Also, D-002 decreased the content of MDA and PCG, the generation of hydroxyl radicals and MPO activity as compared to the positive controls. The highest dose of D-002 (100 mg/kg) increased significantly GSHPX and SOD activities, while all doses used increased CAT activities. Omeprazole $20 \mathrm{mg} /$ $\mathrm{kg}$, the reference drug, reduced significantly the ulcers (93\%), MDA and PCG, the generation of hydroxyl radicals and MPO activity, and increased the CAT, GSH-PX and SOD activities. D-002 treatment produced gastroprotective effects against indomethacin-induced gastric ulceration, which can be related to the reduction of hydroxyl radical generation, lipid peroxidation, protein oxidation and MPO activity, and to the increase of the antioxidant enzymes activities in the rat gastric mucosa. 
Public health services, an essential determinant of health during crisis. Lessons from Cuba, 1989-2000. De Vos P, García A, Alvarez A, Rodríguez A, Bonet M, Van der Stuyft P. Trop Med Int Health. 2012 Feb. Epub ahead of print.

During the 1990s, Cuba was able to overcome a severe crisis, almost without negative health impacts. This national retrospective study covering the years 1989-2000 analyses the country's strategy through essential social, demographic, health process and health outcome indicators. Gross domestic product (GDP) diminished by 34.76\% between 1989 and 1993. In 1994 slow recuperation started. During the crisis, public health expenses increased. The number of family doctors rose from 9.22 to 27.03 per 104 inhabitants between 1989 and 2000. Infant mortality rate and life expectancy exemplify a series of health indicators that continued to improve during the crisis years, whereas low birth weight and tuberculosis incidence are among the few indicators that suffered deterioration. GDP is inversely related to tuberculosis incidence, whereas the average salary is inversely related to low birth weight. Infant mortality rate has a strong negative correlation with the health expenses per inhabitant, the number of maternal homes, the number of family doctors and the proportion of pregnant women receiving care in maternal homes. Life expectancy has a strong positive correlation with health expenses, the number of nursing personnel and the number of medical contacts per inhabitant. The Cuban strategy effectively resolved health risks during the crisis. In times of serious socio-economic constraints, a well conceptualized public health policy can play an important role in maintaining the overall well-being of a population.

Quality of Life of People with HIVIAIDS Receiving Antiretroviral Therapy in Cuba: A
Cross-Sectional Study of the National Population. Aragonés-López C, Pérez-Ávila J, Smith Fawzi MC, Castro A. Am J Public Health. 2012 May;102(5):884-92.

Objectives We studied the effect of antiretroviral therapy (ART) on the quality of life (QOL) of Cubans with HIVIAIDS. Methods We conducted a cross-sectional study including administration of the Medical Outcomes Study-HIV Health Survey Questionnaire to a representative sample of the 1592 Cubans receiving ART in 2004. For univariate analyses, we compared mean HIV scale scores. We used logistic regression models to estimate the association between role function and year of diagnosis, between pain and sex, and between health transition and region of diagnosis, with adjustment for demographics, ART regimen, and clinical status. Results There were 354 participants (73 women, 281 men). Scores for all functional activities showed means higher than 80 out of 100 . Pain interfered more in women than in men (73.2 vs 81.9; $p=0.01$ ). When HIV diagnosis occurred after 2001, the probability of experiencing difficulties performing work (odds ratio $[\mathrm{OR}]=4.42$; $95 \% \mathrm{Cl}=1.83,10.73)$ and pain $(\mathrm{OR}=1.70 ; 95 \%$ $\mathrm{Cl}=1.01,2.88)$ increased compared with earlier diagnosis. People treated with indinavir showed a greater perception of general health (58.9 vs 52.4; $\mathrm{p}=0.045)$ and greater health improvement (78.6 vs 67.8; $p=0.002$ ). Conclusions Although Cubans receiving ART are maintaining a high $\mathrm{QOL}$, we observed significant differences by sex and time of diagnosis. QOL assessment can serve as a health outcome and may allow identification of QOL reductions potentially related to ART side effects.

Very small size proteoliposomes derived from Neisseria meningitidis: An effective adjuvant for antigen-specific cytotoxic $\mathbf{T}$ lymphocyte response stimulation under leukopenic conditions. Oliver L, Fernández $A$, Raymond J, López-Requena A, Fernández LE, Mesa C. Vaccine. 2012 Apr 19;30(19):2963-72.

Leukopenia is a severe condition resulting from both pathological processes and some treatments, like chemotherapy in cancer patients. However, the activation of the patient immune system is required for the success of immunotherapeutic strategies, as cancer vaccines. In this regard, leukopenia constitutes a major hurdle to overcome, mainly due to the impairment of cytotoxic T lymphocyte (CTL) responses. Adjuvants are basic components of vaccine formulations, which might be useful to stimulate immunity under this immunosuppressed condition. To this aim, we tested the capacity of a novel nanoparticulated complex, very small size proteoliposomes (VSSP), to promote CTL even in a leukopenic scenario. Noteworthy, we observed that a VSSP-based OVA vaccine induced a normal antigen-specific CTL response in mice rendered leukopenia by the administration of high doses of the chemotherapeutic agent cyclophosphamide (CY), while under the same conditions the OVA antigen formulated in the TLR-3 agonist polyinosinic-polycytidylic acid $(\mathrm{P}(\mathrm{I}: \mathrm{C}))$ was ineffective. Moreover, an appropriate combination of VSSP with the $\mathrm{P}(\mathrm{I}: \mathrm{C})$ vaccine was able to restore the CD8(+) T cell effector function in leukopenic mice. VSSP induced not only a faster repopulation of immune cells in CY-receiving animals, but also enhanced the recovery of memory $T$ lymphocytes and myeloid dendritic cells (DCs) while simultaneously abrogated the immunosuppressive capacity of myeloid-derived suppressor cells (MDSCs). Our results suggest that VSSP could be a particularly suitable immunomodulator to be used in CTL-promoting active immunotherapy strategies operating in severe immune compromised scenarios. $-1 /$ - 